Research paper

\title{
Impact of anti-PLK1 siRNA-containing F3-targeted liposomes on the viability of both cancer and endothelial cells
}

\author{
Lígia C. Gomes-da-Silva ${ }^{\text {a,b }}$, José S. Ramalho ${ }^{c}$, Maria C. Pedroso de Lima ${ }^{\text {a,d }}$, Sérgio Simões ${ }^{\text {a,b }}$, \\ João N. Moreira ${ }^{a, b, *}$ \\ ${ }^{a}$ CNC - Center for Neurosciences and Cell Biology, University of Coimbra, Portugal \\ ${ }^{\mathrm{b}}$ FFUC - Faculty of Pharmacy, University of Coimbra, Portugal \\ ${ }^{\mathrm{c}}$ Laboratory of Cellular and Molecular Biology, Faculty of Medical Sciences, New University of Lisbon, Portugal \\ ${ }^{\mathrm{d}}$ Department of Life Sciences, Faculty of Sciences and Technology, University of Coimbra, Portugal
}

\section{A R T I C L E I N F O}

\section{Article history:}

Received 19 November 2012

Accepted in revised form 9 April 2013

Available online 7 May 2013

\section{Keywords:}

Nanotechnology

SiRNA

PLK1

Gene silencing

Paclitaxel

Prostate cancer

\begin{abstract}
A B S T R A C T
We have previously described the development of novel sterically stabilized F3-targeted pH-sensitive liposomes, which exhibited the ability to target both cancer and endothelial cells. Herein, the therapeutic potential of those liposomes was assessed upon encapsulation of a siRNA against a well-validated molecular target, PLK1.

Treatment of prostate cancer (PC3) and angiogenic endothelial (HMEC-1) cells with F3-targeted liposomes containing anti-PLK1 siRNA resulted in a significant decrease in cell viability, which was mediated by a marked PLK1 silencing, both at the mRNA and protein levels. Furthermore, pre-treatment of PC3 cells with F3-targeted liposomes containing anti-PLK1 siRNA enabled a 3-fold reduction of paclitaxel IC $_{50}$ and a 2.5-fold augment of the percentage of cancer cells in G2/mitosis arrest, which ultimately culminated in cell death.

Overall, the F3-targeted nanocarrier containing an anti-PLK1 siRNA might constitute a valuable system for prostate cancer treatment, either applied in a single schedule or combined with conventional chemotherapy.
\end{abstract}

(c) 2013 Elsevier B.V. All rights reserved.

\section{Introduction}

Prostate cancer is a highly prevalent disease and the second most common cancer among males in developed countries, being the sixth leading cause of cancer-related death in men [1]. Conventional anti-prostate cancer therapies include surgery, hormonal ablation, radiation, immunotherapy, and chemotherapy. Despite increasing efforts, these therapies are yet to be effective in patients with locally advanced and/or metastatic disease [2]. Moreover, current available chemotherapies often result in dose-limiting side

Abbreviations: PLK1, polo-like kinase 1; siRNA, small-interfering RNA; CTR, control; PC3, PC3 prostate cancer cells; HMEC-1, human microvascular endothelial cells; eGFP, enhanced Green Fluorescent Protein; $\mathrm{IC}_{50}$, half maximal death concentration; DRI, dose reduction index; G2/M phases, G2/mitosis phases; DOTAP, 1, 2dioleoyl-3-trimethylammonium-propane; DSPC, 1,2-distearoyl-sn-glycero-3-phosphocholine; DOPE, 1,2-dioleoyl-sn-glycero-3-phosphoethanolamine; $\mathrm{C}_{16}$-Ceramide $\mathrm{mPEG}_{2000}$, N-Palmitoyl-Sphingosine-1-[Succinyl(MethoxyPolyethylene Glycol $)_{2000}$ ]; DSPE-PEG-MAL, 12-Distearoyl-sn-Glycero-3-Phosphoethanolamine-N[Maleimide (Polyethylene Glycol) 2000 ] ammonium salt; CHOL, cholesterol; CHEM, Scholesteryl hemisuccinate; PBS, phosphate buffer saline; SD, standard deviation.

* Corresponding author. Center for Neurosciences and Cell Biology, University of Coimbra, Largo Marquês de Bombal, 3004-517 Coimbra, Portugal. Tel.: +351 916885272.

E-mail address: jmoreira@ff.uc.pt (J.N. Moreira). effects due to unspecific accumulation in healthy organs. Additionally, induction of resistance to the chemotherapeutic agents is one of the main reasons for treatment failures $[3,4]$.

The better understanding of the different signaling pathways of cancer cells and supportive stroma has allowed the identification of numerous molecular targets whose downregulation can lead to cell death, angiogenesis inhibition, or reversion of the malignant phenotype [5,6]. This broad and deep knowledge provides the basis for the rational design of novel therapeutic strategies envisaging the modulation, at different cell levels within a tumor, of specific signaling pathways that dictate tumor growth and aggressiveness. Several reports have demonstrated that increased sensitivity to chemotherapy can be achieved by targeting relevant molecular targets [7-9]. With this strategy, it is envisaged that improved clinical outcomes will be achieved with lower drug doses and consequent reduction of unwanted side effects. Furthermore, considering the biological heterogeneity of a tumor, it is expected that a multi-targeted therapy at the intracellular level may produce better therapeutic results.

Polo-like kinase 1 (Plk1) is a serine/threonine kinase that regulates mitotic entry and progression. Its expression level in normal tissues is very low or undetectable, but is overexpressed in a wide variety of tumors [10], including prostate cancer [11], being often 
correlated with poor patient prognosis [12-14]. Additionally, some studies have suggested Plk1 as a marker of high risk of metastasis formation $[15,16]$. Plk1 overexpression in cancer cells enables the overriding of the cell cycle checkpoints, thus contributing for the capability of cancer cells to proliferate in an uncontrolled manner, even when DNA damage is present, which promotes chromosome instability and aneuploidy [17]. It has been demonstrated that downregulation of Plk1 leads to growth inhibition, mitotic arrest and induction of apoptosis in cancer cell lines from different histological origins [11,18-20], and in vivo suppression of tumor growth [19-22]. In contrast, Plk1 depletion in normal cells has not revealed to be harmful, thus suggesting that treatment specificity can be achieved when therapies that specifically modulate Plk1 are applied [20]. Recently, Plk1 downregulation was also correlated with a significant decrease in the angiogenic network of a mouse model of human colorectal carcinoma, thus indicating that Plk1 can have a pro-angiogenic effect [19]. Overall, Plk1 is a well-validated anti-cancer therapeutic target that might have impact at two different levels within prostate cancer: the cancer cells and the endothelial cells from angiogenic blood vessels.

One promising strategy to mediate gene downregulation is undoubtly throughout RNA-interference molecules such as smallinterfering RNAs (siRNAs) [23,24]. However, the in vivo application of these molecules faces numerous barriers such as degradation by blood nucleases, internalization by the mononuclear phagocytic system, rapid elimination by the kidneys, and limited internalization by the target cells [25-27]. Therefore, their therapeutic application is essentially dependent on the development of nanocarriers that can mediate efficient siRNA delivery into the target cells.

We have previously described the development of sterically stabilized F3-targeted $\mathrm{pH}$-sensitive liposomes fulfilling the physico-chemical requisites demanded to a nanocarrier for systemic delivery of siRNA [28]. As the F3 peptide is specifically internalized by nucleolin, a receptor overexpressed on the surface of cancer and endothelial cells from angiogenic tumor blood vessels [29], the developed F3-targeted liposomes were internalized by both breast cancer and HMEC-1 endothelial cells [28]. The developed F3-targeted $\mathrm{pH}$-sensitive liposomes incorporate in their composition the fusogenic lipid 1,2-dioleoyl-sn-glycero-3-phosphoethanolamine (DOPE), which has an inverted cone-shape, and its stabilizer cholesteryl hemisuccinate (CHEMS). At physiological $\mathrm{pH}$, an organized and stable lipid bilayer is formed. However, under the acidic conditions of the endocytic pathway, CHEMS is protonated, loosing its ability to stabilize the inverted cone-shape of DOPE and thus leading to the destabilization of the liposomal membrane. Such destabilization favors the release of the encapsulated siRNA into the cell cytoplasm, which was indeed correlated with an efficient silencing of a reporter gene (enhanced Green Fluorescent Protein, eGFP) [28]. In addition, the developed $\mathrm{F} 3$-targeted $\mathrm{pH}$-sensitive liposomes were stable and biocompatible with blood and no signs of toxicity were observed upon multiple systemic administrations [28].

Therefore, the goal of this work was to evaluate the potential of the previously developed dual-targeted nanoplatform for the delivery of siRNA against a relevant therapeutic target (PLK1) to prostate cancer and endothelial cells, and its role in the chemosensitization of cancer cells to paclitaxel.

\section{Materials and methods}

\subsection{Materials}

1,2-Dioleoyl-3-trimethylammonium-propane (DOTAP), 1,2-distearoyl-sn-glycero-3-phosphocholine (DSPC), 1,2-dioleoyl-sn-glycero3-phosphoethanolamine (DOPE), N-Palmitoyl-Sphingosine-1-[Succinyl(MethoxyPolyethylene Glycol $)_{2000}$ ( $\left(\mathrm{C}_{16}\right.$-Ceramide $\left.\mathrm{mPEG}_{2000}\right)$,
1,2-Distearoyl-sn-Glycero-3-Phosphoethanolamine-

N-[Maleimide (Polyethylene Glycol) 2000 ] ammonium salt (DSPEPEG-MAL), and L- $\alpha$-Phosphatidylethanolamine-N-(lissamine rhodamine B sulfonyl) (Rho-PE) were purchased from Avanti Polar Lipids (USA). The lipids, cholesterol (CHOL) and cholesteryl hemisuccinate (CHEMS), were obtained from Sigma (Germany).

The anti-PLK1 siRNA (antisense sequence 5'-GUAAAGAA CUCGUCAUUAAGCdTdT- $3^{\prime}$ ) and the non-specific control siRNA (CTR siRNA) (antisense sequence 5'-ACGUGACACGUUCGGAGAAdTdT$3^{\prime}$ ) were synthesized by Dharmacon (USA). The F3 peptide (KDEPQRRSARLSAKPAPPKPEPKPKKAPAKK) and the non-specific (NS) peptide (ARALPSQRSR) were synthesized by Genecust (Luxembourg).

\subsection{Cell lines}

The human prostate cancer cell line, PC3, was purchased from American Type Culture Collection (ATCC). The human microvascular endothelial cell line, HMEC-1, was a generous gift from the Center for Disease Control and Prevention (USA). PC3 cells were cultured in RPMI 1640 medium (Sigma, Germany) supplemented with $10 \%(\mathrm{v} / \mathrm{v})$ heat-inactivated fetal bovine serum (Invitrogen, USA), $100 \mathrm{U} / \mathrm{ml}$ penicillin, and $100 \mu \mathrm{g} / \mathrm{ml}$ streptomycin (Invitrogen, USA). HMEC- 1 cells were cultured in RPMI 1640 supplemented with $10 \mathrm{ng} / \mathrm{ml}$ of mouse epidermal growth factor (mEGF) (Sigma, Germany) and $1 \mu \mathrm{g} / \mathrm{ml}$ hydrocortisone (Sigma, Germany). Cells were maintained at $37{ }^{\circ} \mathrm{C}$, in a $90 \%$ humidified atmosphere, containing $5 \% \mathrm{CO}_{2}$. Cells were maintained in the exponential growth phase and used at a low passage number. All the experiments involving the mentioned cells were conducted in medium containing $10 \%$ of serum.

\subsection{Preparation of sterically stabilized liposomes}

Encapsulation of siRNA into sterically stabilized liposomes was preformed as previously described [28]. Briefly, a lipid mixture of DOTAP/DSPC/DOPE/CHOL/CHEMS/CerC ${ }_{16} \mathrm{PEG}_{2000}$, (25:10:25:26:10:4 $\mathrm{mol} \%$, relative to total lipid) in absolute ethanol was added to the anti-PLK1 siRNA, or the control (CTR) siRNA, in $20 \mathrm{mM}$ citrate buffer, pH 6, previously heated at $65^{\circ} \mathrm{C}$.

The resulting particles were then extruded 21 times through polycarbonate membranes of $100 \mathrm{~nm}$ pore diameter, using a LiposoFast basic extruder (Avestin, Canada). Removal of ethanol and non-encapsulated siRNA was carried out upon running the extruded nanoparticles through a Sepharose CL-4B column equilibrated with HEPES buffered saline (HBS) (20 mM HEPES, $145 \mathrm{mM}$ $\mathrm{NaCl}$ ), $\mathrm{pH}$ 7.4. Targeted liposomes were prepared by the post-insertion method [30]. Briefly, thiolated F3 peptide or NS peptide were first coupled to DSPE-PEG-MAL micelles, prepared in MES buffered saline (MBS) (20 mM MES, $20 \mathrm{mM}$ HEPES), pH 6.5. Insertion of DSPE-PEG-MAL-F3 or DSPE-PEG-MAL-NS conjugates onto the preformed sterically stabilized liposomes took place upon incubation of the latter with the corresponding micelles at $50{ }^{\circ} \mathrm{C}$ for $1 \mathrm{~h}$. For the non-targeted lipid particles, post-insertion was performed only with plain DSPE-PEG-MAL micelles.

The resulting F3-targeted liposomes were characterized by a mean size of $146.13 \pm 2.96 \mathrm{~nm}$, a polydispersity index of 0.148 \pm 0.05 , a zeta potential close to the neutrality $(-1.94 \pm 7.06 \mathrm{mV})$, and an encapsulation efficiency of $75.80 \pm 15.07 \%$. Freeze-fracture electron microscopy clearly indicated that the F3-targeted liposomes containing the anti-PLK1 siRNA were spherical vesicles (data not shown). 


\subsection{Assessment of cellular association by flow cytometry}

Two hundred and fifty thousand of PC3 cells were seeded in 48well plates. Twenty-four hours later, cells were incubated at $37^{\circ} \mathrm{C}$ during $1 \mathrm{~h}$, with rhodamine-labeled F3-targeted, NS-targeted, or non-targeted liposomes at $0.2,0.4$, or $0.6 \mathrm{mM}$ of total lipid. Afterward, cells were washed three times with phosphate buffer saline (PBS) pH 7.4, detached, and immediately analyzed by flow cytometry using a FACS Calibur flow cytometer (BD, Biosciences). Rhodamine fluorescence was evaluated in the FL2 channel, and a total of 20.000 events were collected. Data were analyzed with the Cell Quest Pro software.

\subsection{Assessment of cell viability}

In order to evaluate the impact on cell viability of different liposomal formulations containing anti-PLK1 siRNA, 25.000 PC3 prostate cancer cells were seeded in 24-well plates. Twenty-four hours later, cells were transfected with either anti-PLK1 siRNA (100 nM to $2 \mu \mathrm{M})$ encapsulated in F3-targeted or non-targeted liposomes, or with the CTR siRNA encapsulated in the former, at $37{ }^{\circ} \mathrm{C}$ during $8 \mathrm{~h}$. Afterward, the culture medium was replaced by fresh medium and a second transfection was performed $40 \mathrm{~h}$ after the first treatment. Ninety-six hours after the beginning of the experiment ( $40 \mathrm{~h}$ after the second transfection), cell viability was evaluated using the Resazurin reduction method. Briefly, cells were incubated, at $37^{\circ} \mathrm{C}$ during $2 \mathrm{~h}$, with $10 \%$ of resazurin dye in culture medium, followed by absorbance measurements at $540 \mathrm{~nm}$ (reduced form) and $630 \mathrm{~nm}$ (oxidized form) in a microplate fluorimeter Microdevices SpectraMax Gemini EM. Cell viability was extrapolated from the resazurin reduction using the following equation: [ $\left(\varepsilon_{\text {oxi630 }} * A 540-\varepsilon_{\text {oxi540 }}\right.$ $\left.* A 630)_{\text {treated cells }} /\left(\varepsilon_{\text {oxi630 }} * A 540-\varepsilon_{\text {oxi540 }} * A 630\right)_{\text {untreatedcells }}\right] * 100$.

\subsection{Quantification of PLK1 mRNA by quantitative real-time reverse- transcription PCR ( $q R T-P C R)$}

Using the same experimental conditions mentioned in the previous section, PLK1 mRNA was assessed $24 \mathrm{~h}$ after the second transfection ( $72 \mathrm{~h}$ after the beginning of the experiment) by qRTPCR.

Cells were harvested and RNA extracted with Quick-RNA MicroPrep (Zymo Research, Germany) according to the manufacturer's instructions. The Quanti-iTTM Ribogreen (Invitrogen, USA) reagent was used to quantify the extracted RNA. Then, $0.5 \mu \mathrm{g}$ of RNA was reversed transcribed into cDNA using the QuantiTec Reverse Transcription kit (Qiagen, Germany) following manufacturer's instructions.

Quantitative real-time PCR ( $\mathrm{qPCR}$ ) was performed using the Iq ${ }^{\mathrm{TM}}$ SYBR Green Supermix (Bio-Rad, USA). Beta-2-microglobulin $(\beta 2 M)$ was used as the endogenous control (housekeeping gene). The forward primer for $\beta 2 M$ was $5^{\prime}$-GAGTATGCCTGCCGTGTG-3' and the reverse primer was 5'-AATCCAAATGCGGCATCT-3' (Microsynth, Switzerland). For PLK1, the QuantiTect ${ }^{\mathrm{TM}}$ Primer Assay (Qiagen, Germany) was used.

The optimized qPCR conditions included the activation of DNA HotStartTaq Plus DNA polymerase followed by 40 cycles of two steps: a first step of denaturation $\left(10 \mathrm{~s}\right.$ at $\left.95^{\circ} \mathrm{C}\right)$ and a second step of combined annealing/extension $\left(30 \mathrm{~s}\right.$ at $60^{\circ} \mathrm{C}$ ). After the qPCR, a melting curve analysis of the PCR products was performed to confirm their specificity.

The threshold cycle $(\mathrm{Ct})$ values were generated by the iQ5 Optical System Software. The level of PLK1 mRNA was calculated by the Livak method, $2^{-\Delta \Delta \mathrm{Ct}} \times 100$, where $\Delta \Delta \mathrm{Ct}=(\mathrm{Ct} \quad$ PLK1 $-\mathrm{Ct}$ $\beta 2 \mathrm{M})_{\text {treated }}-(\mathrm{Ct} \text { PLK1 }-\mathrm{Ct} \beta 2 \mathrm{M})_{\text {untreated }}$. The application of this method relies on the similar PCR efficiencies between the target gene and the housekeeping gene.

\subsection{Evaluation of Plk1 protein by Western blot}

Using the same experimental conditions mentioned in the previous section, Plk1 protein was evaluated by Western blot $48 \mathrm{~h}$ after the second transfection ( $96 \mathrm{~h}$ after the beginning of the experiment).

Protein extracts were obtained from PC3 cells using a lysis buffer (10 mM Tris, 150 mM NaCl, 5 mM EDTA, 1\% (v/v) Triton X-100) containing a protease inhibitor cocktail (Roche, USA) and submitted to 3 freeze-thaw cycles. After centrifugation, protein content was separated and quantified using the $\mathrm{BCA}^{\mathrm{TM}}$ Protein Assay Kit (Pierce, USA). Then, $20 \mu \mathrm{g}$ of total protein was resuspended in loading buffer $(0.0625 \mathrm{mM}$ Tris $\mathrm{HCl}, 25 \%$ glycerol, $4 \%$ sodium dodecyl sulfate, $0.01 \%$ bromophenol blue, $5 \%$ beta-mercaptoethanol), incubated for $5 \mathrm{~min}$ at $95{ }^{\circ} \mathrm{C}$ and loaded onto a $10 \%$ resolving SDS-polyacrylamide gel. After electrophoresis $(100 \mathrm{~V}$ for approximately $1.5 \mathrm{~h}$ ), proteins were transferred onto a polyvinylidene difluoride (PVDF) membrane (GE Healthcare Amersham Hybond ${ }^{\mathrm{TM}}-\mathrm{P}, \mathrm{UK}$ ), which had been previously incubated with a blocking solution containing $5 \%$ of non-fat milk, during $1 \mathrm{~h}$ at room temperature. The membrane was further incubated with a monoclonal antibody anti-Plk1 (Sigma, Germany), overnight at $4{ }^{\circ} \mathrm{C}$, followed by incubation with the appropriate secondary antibody linked to alkaline phosphatase (GE Healthcare, Sweden), for $1 \mathrm{~h}$ at room temperature. After incubation, the membrane was washed and incubated with ECF (alkaline phosphatase substrate) (GE Healthcare, Sweden) for $5 \mathrm{~min}$. The protein bands were detected by chemifluorescence in a VersaDoc Imaging System (Bio-Rad, USA). The analysis of band intensity was performed using the Quantity One software (Bio-Rad, USA).

Equal protein loading was shown by reprobing the membrane with an anti-actin antibody (Sigma, Germany) and with the appropriate secondary antibody.

\subsection{Sensitization of PC3 cells to paclitaxel}

Twenty-five thousand PC3 cells were seeded in 48-well plates and transfected $24 \mathrm{~h}$ later with either anti-PLK1 siRNA encapsulated in F3-targeted or non-targeted liposomes, or with the CTR siRNA encapsulated in the former, at a final siRNA concentration of $1 \mu \mathrm{M}$. After $8 \mathrm{~h}$ of incubation at $37^{\circ} \mathrm{C}$, the cultured medium was replaced by fresh medium.

Incubation with paclitaxel was performed $40 \mathrm{~h}$ after the transfection with the developed liposomes, for $24 \mathrm{~h}$ at $37^{\circ} \mathrm{C}$. The cell culture medium was then replaced with fresh one, and at $96 \mathrm{~h}$, cell viability was assessed by the resazurin reduction method, as previously described. In order to evaluate the impact of the sequential treatment in the overall cell viability, control cells were solely incubated with culture medium (untreated cells).

The required paclitaxel concentration to promote $50 \%$ reduction of cell viability $\left(\mathrm{IC}_{50}\right)$ was determined by a non-linear regression assuming sigmoidal dose-response. The paclitaxel's dose reduction index (DRI) achieved upon pre-incubation with the different liposomal formulations was based on the ratio of $\mathrm{IC}_{50}$ of paclitaxel determined when used alone and when combined with the liposomes.

\subsection{Cell cycle analysis by flow cytometry}

The effect of liposomal anti-PLK1 siRNA and/or paclitaxel on cell cycle distribution of PC 3 cells was evaluated, using the same experimental conditions mentioned in the previous section. After incubation, cancer cells were fixed in $70 \%$ ethanol during $2 \mathrm{~h}$ at $4{ }^{\circ} \mathrm{C}$ and then washed with PBS and labeled with 7-amino-actinomycin $\mathrm{D}$ (7-AAD) (Sigma, Germany) at $4{ }^{\circ} \mathrm{C}$ for $30 \mathrm{~min}$. In the analysis performed by flow cytometry using a FACS Calibur flow cytometer 
(BD, Biosciences), a total of 50.000 events were collected and 7AAD fluorescence was evaluated in the FL3 channel. Cell cycle analysis was performed with ModFit LT software.

\subsection{Statistical analysis}

The results are presented as the mean \pm standard deviation (SD) of at least 3 independent experiments, each one with triplicates. Two-way ANOVA with Bonferroni's post-test was used to determine statistically significant differences of the means between multiple groups. Statistical differences were presented at probability levels of $p>0.05, p<0.05, p<0.01$ and $p<0.001$.

\section{Results}

\subsection{Cellular association studies}

Within the range of the tested concentrations, the attachment of the F3 peptide to the liposomal surface led to a 7- to 11-fold improvement on the extent of PC3 cellular association, when compared with the non-targeted liposomes or liposomes targeted by a non-specific peptide. The extent of cellular association for the F3targeted liposomes was clearly dependent on the lipid concentration (Fig. 1A). The narrow shape of the histograms generated by flow cytometry demonstrated that the F3-targeted liposomes bound to the bulk PC3 cells in a similar extent, indicating that a homogenous cellular association was achieved (Fig. 1B).

\subsection{Effect of F3-targeted liposomes containing an anti-PLK1 siRNA on cancer and endothelial cells viability}

Plk1 has been suggested as a promising molecular target since its downregulation results in mitosis arrest, proliferation inhibition, cancer cell death, as well as in vivo tumor growth inhibition [10]. Therefore, aiming at evaluating the therapeutic potential of the developed F3-targeted nanotechnology-based strategy, a siRNA against PLK1 was designed and encapsulated in the mentioned liposomes.

Cell proliferation studies demonstrated that F3-targeted liposomes, entrapping the anti-PLK1 siRNA, produced a significant anti-proliferative effect and in a dose-dependent manner in both prostate cancer (Fig. 2A) and endothelial cells from angiogenic blood vessels (Fig. 2B). At $2 \mu \mathrm{M}$ of anti-PLK1 siRNA, PC3 and HMEC-1 cell viability decreased $57.0 \pm 9.03 \%$ and $39.4 \pm 9.86 \%$, respectively. On the other hand, the non-targeted counterpart did not have significant effects on the viability of both those cells, which is in accordance with its residual cellular association previously observed. Taken together, these results have clearly demonstrated that the presence of the F3 peptide on the liposomal surface strongly contributed to improve the cytotoxicity of an anti-PLK1 siRNA, through its ability to mediate an effective interaction with the target cells. In the case of HMEC- 1 cells, the observed effect encompassed a certain extent of non-specific effects, as revealed by the experiments carried out with F3-targeted liposomes containing the control siRNA (Fig. 2B). This result is in accordance with other reports, where it was demonstrated that different siRNA sequences, regardless of their target, can have anti-angiogenic effects [31,32].

\subsection{Assessment of PLK1 mRNA and protein levels}

In order to confirm that the decrease in PC3 cell viability was due to PLK1 downregulation, the levels of PLK1 mRNA and protein were assessed by qRT-PCR and Western blot, respectively. In fact, following incubation with F3-targeted liposomes entrapping antiPLK1 siRNA, at $100 \mathrm{nM}$ to $2 \mu \mathrm{M}$, a $33.92 \pm 7.60$ to $59.74 \pm 5.07 \%$ PLK1 mRNA reduction was observed, showing that this liposomal formulation was 1.8 - to 2.3 -fold more effective than the non-targeted counterpart. In addition, no significant reduction on the mRNA levels was reached with the control siRNA, indicating that the observed PLK1 downregulation was sequence-specific (Fig. 3A). In parallel experiments, the same trend was observed at the level of protein downregulation. Incubation of PC3 cells with F3-targeted liposomes containing anti-PLK1 siRNA promoted a significant reduction in the protein levels, approximately $25 \%$ at $500 \mathrm{nM}$ and $50 \%$ at $2 \mu \mathrm{M}$ of siRNA (Fig. 3B and C). In contrast, no effect on Plk1 protein level was observed upon incubation with non-targeted liposomes containing anti-PLK1 siRNA or with F3-targeted liposomes entrapping the control siRNA.

\subsection{Sensitization of PC3 cells to paclitaxel upon pre-treatment with F3-targeted liposomes containing an anti-PLK1 siRNA}

The anti-proliferative effect of the sequential treatment of PC3 cells with the developed F3-targeted liposomes containing antiPLK1 siRNA and free paclitaxel was assessed. Free paclitaxel, within the range of $1.56-6.25 \mathrm{nM}$, had an anti-proliferative effect from $2.35 \pm 4.46$ to $24.80 \pm 5.80 \%$. Treatments with F3-targeted liposomes containing anti-PLK1 siRNA, followed by free paclitaxel within the above-mentioned range, reduced cell viability from
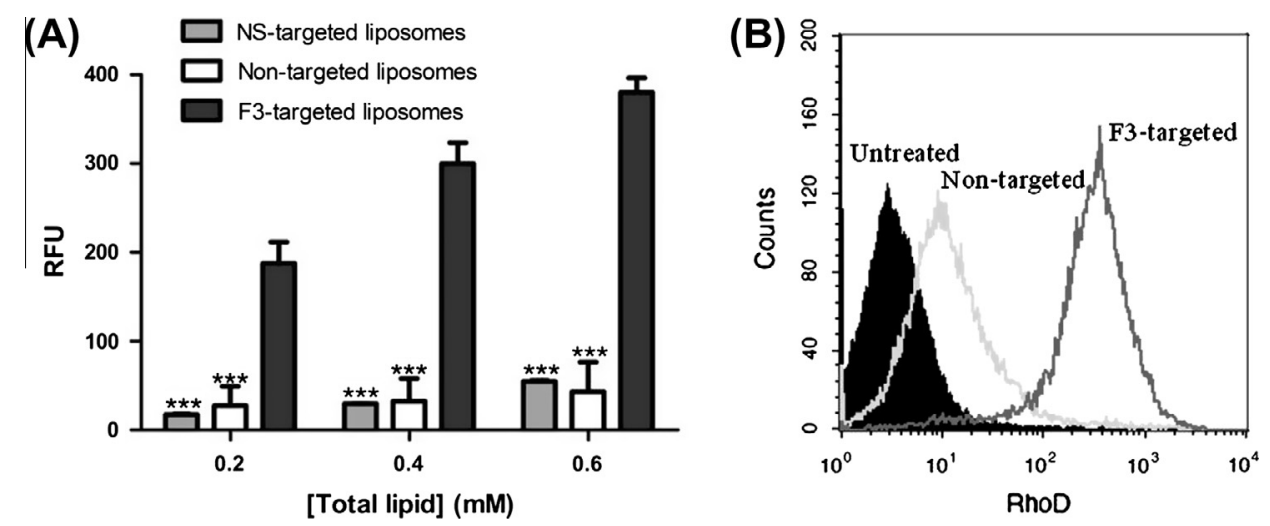

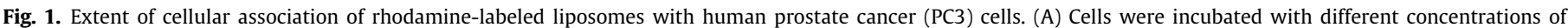

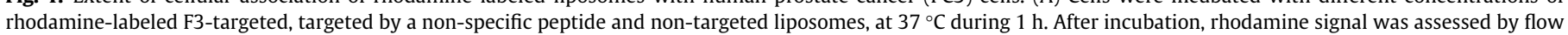

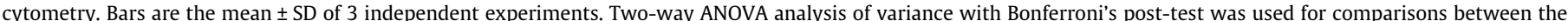

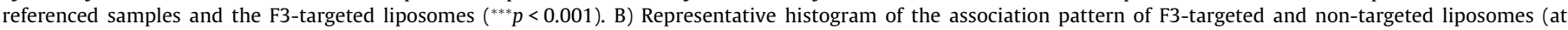
$0.4 \mathrm{mM}$ of total lipid) with PC3 cells, upon incubation at $37^{\circ} \mathrm{C}$ during $1 \mathrm{~h}$. 

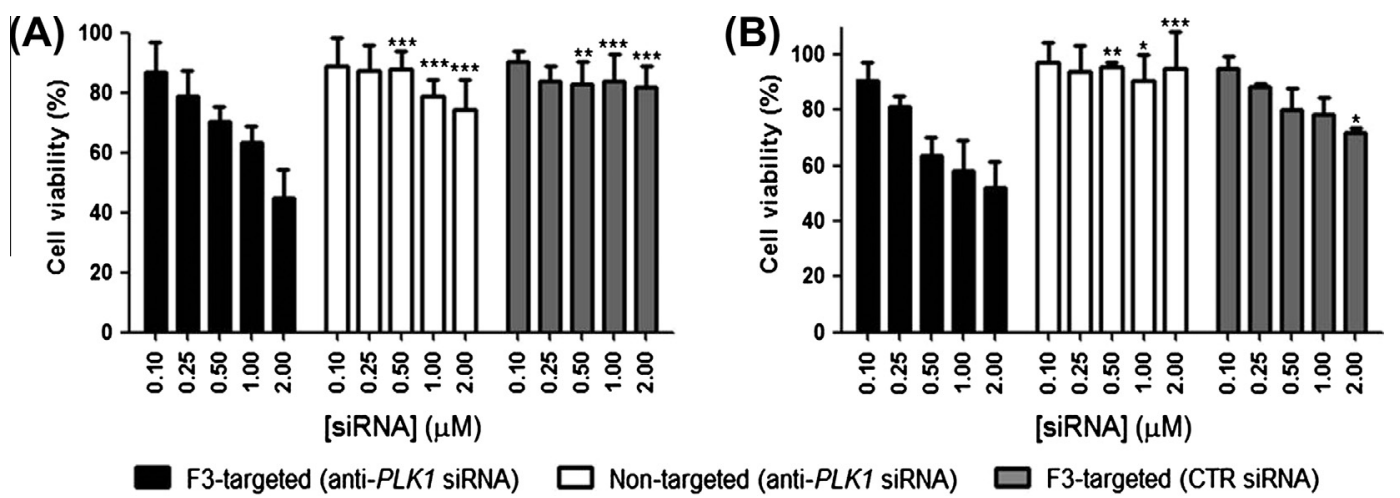

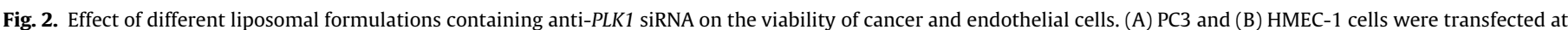

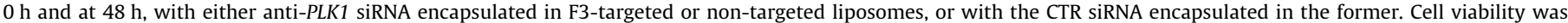

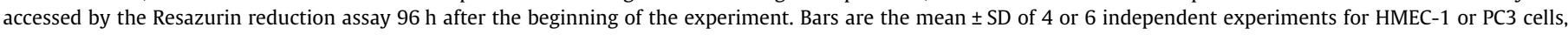

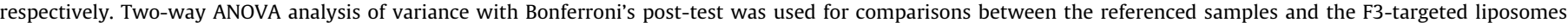
containing the anti-PLK1 siRNA $\left({ }^{* * *} p<0.001\right.$; ${ }^{* *} p<0.01$; no symbol $\left.p>0.05\right)$.
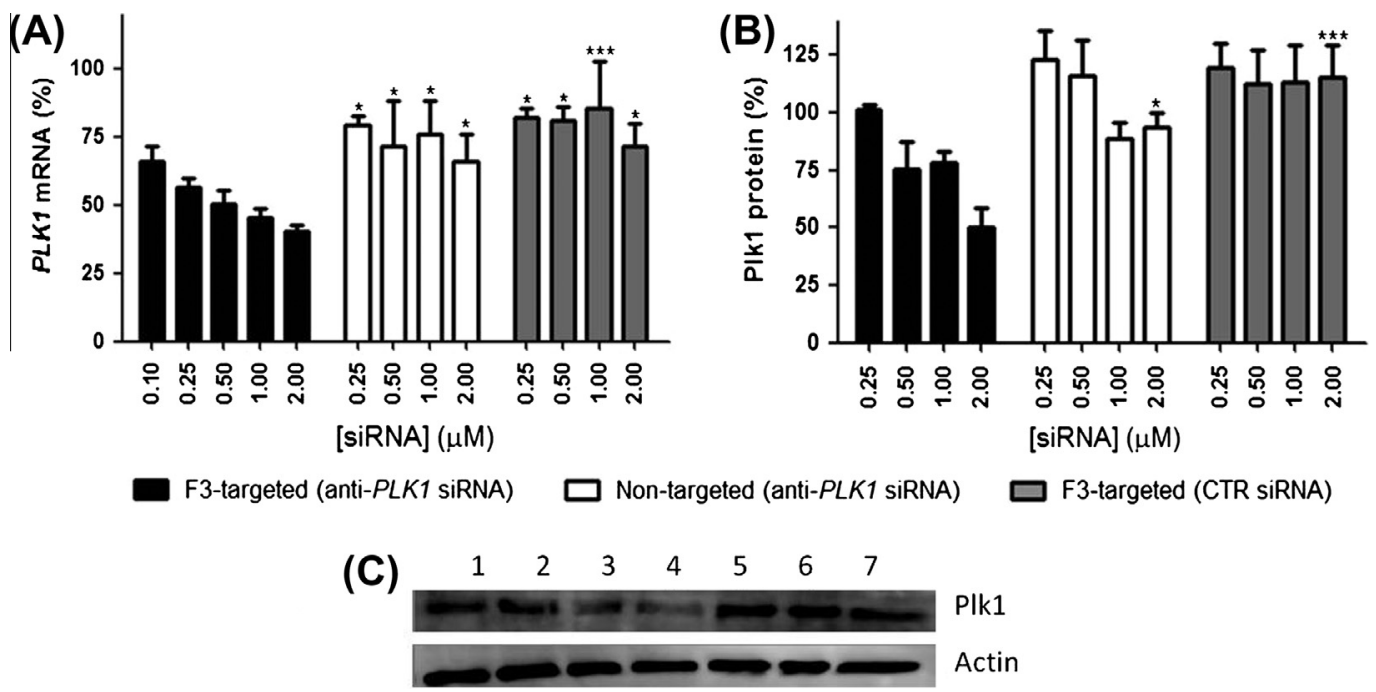

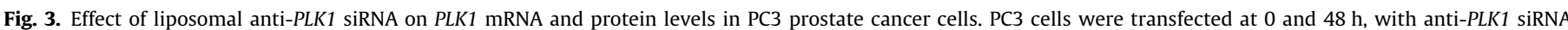

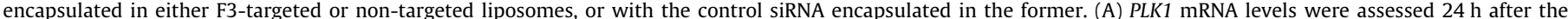

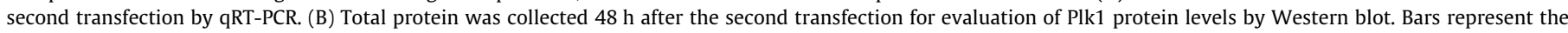

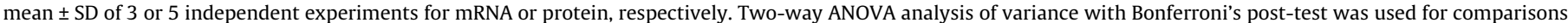

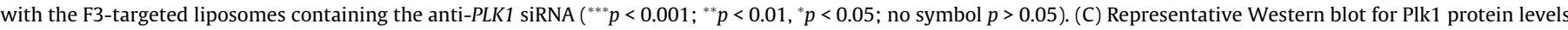

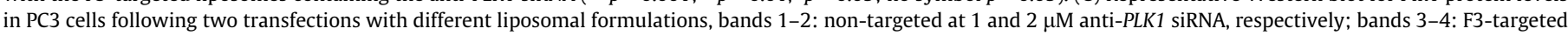
at 1 and $2 \mu \mathrm{M}$ anti-PLK1 siRNA, respectively; band 5-6: F3-targeted at 1 and $2 \mu \mathrm{M}$ CTR siRNA, respectively; and band 7: untreated cells.

$40.09 \pm 11.92$ to $63.07 \pm 5.88 \%$. Sequential treatments with non-targeted liposomes containing anti-PLK1 siRNA or F3-targeted liposomes containing a non-specific siRNA and paclitaxel also enhanced cell death, albeit the differences were not statistically significant when compared with the drug alone $(p>0.005)$. For paclitaxel concentrations higher than $6.25 \mathrm{nM}$, no significant improvements were achieved with the liposomal pre-treatments as a high anti-proliferative effect was already observed upon treatments with only the free drug (Fig. 4).

It was then evaluated whether the sequential treatment with the different liposomal formulations and paclitaxel could actually result in increased paclitaxel's sensitivity. Pre-treatment of PC3 cells with F3-targeted liposomes containing anti-PLK1 siRNA decreased paclitaxel's $\mathrm{IC}_{50}$ from $9.07 \pm 1.58$ to $2.82 \pm 0.58 \mathrm{nM}$. Although reduction of paclitaxel's $\mathrm{IC}_{50}$ was also achieved with pre-treatments with non-targeted liposomes containing antiPLK1 siRNA ( $5.19 \pm 0.54 \mathrm{nM})$ or F3-targeted liposomes containing the non-specific siRNA $(4.92 \pm 0.63 \mathrm{nM})$ (Table 1$)$, the highest DRI
(3.22) was attained with the F3-targeted liposomes containing anti-PLK1 siRNA (Table 1). These results supported our hypothesis that the developed F3-targeted nanotechnology-based strategy entrapping a siRNA targeting a cancer-relevant molecular target might have beneficial effects in oncology, both in single and combined treatments.

\subsection{Cell cycle analysis}

To gain further insights into the cellular effects of the sequential treatments of F3-targeted liposomes entrapping anti-PLK1 siRNA with paclitaxel, cell cycle distribution was analyzed.

Single treatments of PC3 cells with $6.25 \mathrm{nM}$ paclitaxel did not affect the cell cycle distribution, showing $20-30 \%$ of cells in $\mathrm{G}_{2} / \mathrm{M}$ phase (Fig. 5A and D). Pre-treatment with F3-targeted liposomes containing anti-PLK1 siRNA, followed by exposure to the free drug, increased the percentage of cells in $\mathrm{G}_{2} / \mathrm{M}$ phase to $70 \%$ (Fig. $5 \mathrm{~F}$ ), which corresponded to 2.5 - or 3 -fold increase relative to cells 


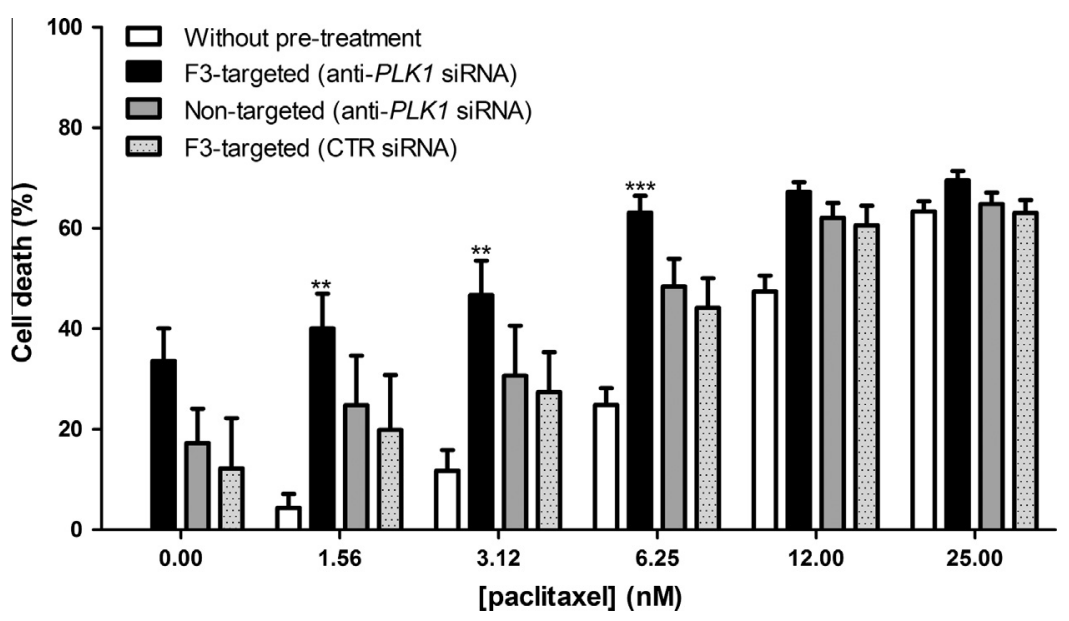

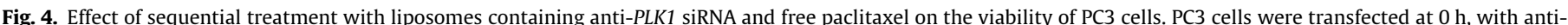

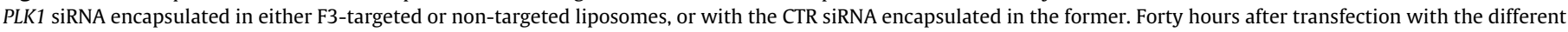

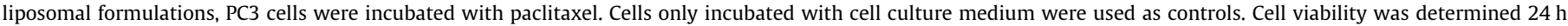

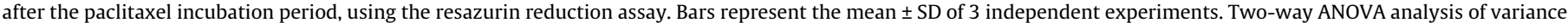

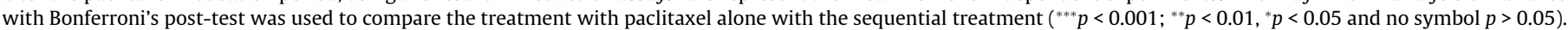

Table 1

Effect of pre-treatments with different liposomal formulations of anti-PLK1 siRNA on paclitaxel's IC ${ }_{50}$ and DRI.

\begin{tabular}{|c|c|c|c|c|}
\hline & Paclitaxel alone & $\begin{array}{l}\text { F3-targeted } \\
(\text { anti-PLK1 siRNA) + paclitaxel }\end{array}$ & $\begin{array}{l}\text { Non-targeted } \\
(\text { anti-PLK1 siRNA) + paclitaxel }\end{array}$ & $\begin{array}{l}\text { F3-targeted } \\
(\text { CTR siRNA) + paclitaxel }\end{array}$ \\
\hline $\mathrm{IC}_{50}(\mathrm{nM})$ & $9.07 \pm 1.58$ & $2.82 \pm 0.58^{\# \# \#}$ & $5.19 \pm 0.54^{\# \# * *}$ & $4.92 \pm 0.63^{\# \# * *}$ \\
\hline DRI & - & 3.22 & 1.75 & 1.84 \\
\hline
\end{tabular}

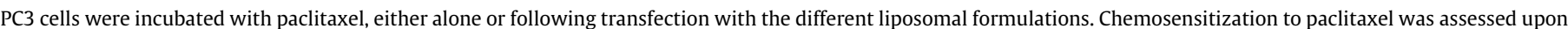

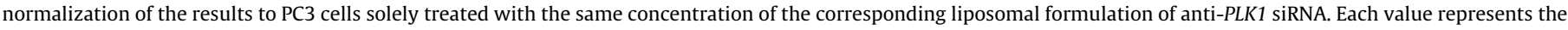
mean \pm SD of 3 independent experiments. One-way ANOVA analysis of variance with Dunnett's post-test was used.

\#\#\# $p<0.001$ when the comparison was between the treatment with paclitaxel alone and the sequential treatments.

\#\# $p<0.01$.

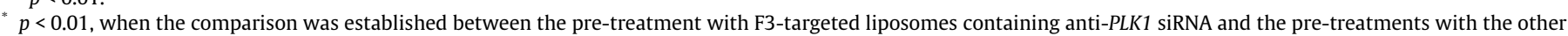
control formulations.

treated solely with paclitaxel or untreated cells, respectively. In contrast, sequential treatments with the non-targeted counterpart resulted only in a minor increase in the number of cells in $\mathrm{G}_{2} / \mathrm{M}$ phase (Fig. 5E). In addition, the same concentration of the control siRNA entrapped in F3-targeted liposomes alone, or in combination with paclitaxel, did not alter the cell cycle distribution significantly (data not shown).

Although it has been described that PLK1 silencing by itself induces cell cycle arrest [11,18], PC3 cells treated solely with F3-targeted liposomes containing anti-PLK1 siRNA did not demonstrate a considerable increase in the $\mathrm{G} 2 / \mathrm{M}$ phase (Fig. $5 \mathrm{C}$ ). This result can be explained by the long time between the liposomal treatment (at time $0 \mathrm{~h}$ ) and the cell cycle evaluation (at $96 \mathrm{~h}$ ), which may have allowed the recovery of Plk1 levels.

\section{Discussion}

Despite prostate cancer death rates have been decreasing in many developed countries due to its detection at early stages as well as improved treatment options, recurrence and formation of distant metastasis are still serious problems that regularly end in morbidity [1]. Moreover, severe side effects and appearance of drug resistance are serious limitations of common chemotherapeutic drugs $[3,4]$. Therefore, novel therapeutic strategies with improved molecular and cellular specificity are urgently needed. In this respect, RNA-interference molecules, such as siRNA, might constitute a new strategy for cancer treatment taking advantage of their ability to downregulate gene expression.

However, their translation to the clinical set has been limited by the lack of a proper delivery system capable of mediating effective delivery to distant sites of disease, as solid tumors, following intravenous administration [25-27]. As an attempt to overcome this major drawback, we have previously developed a F3-targeted pH-sensitive lipid-based nanoparticle for siRNA delivery toward cancer and endothelial cells from angiogenic tumor blood vessels [28]. Aiming at assessing the true therapeutic relevance of the developed targeted nanosystem, an anti-PLK1 siRNA was designed and encapsulated in the former.

Cellular association studies showed that the F3-targeted liposomes were efficiently internalized by PC3 cells in a homogenous manner (Fig. 1), which is in accordance with the internalization pattern observed for these F3-targeted liposomes in both human breast cancer and endothelial cells from angiogenic blood vessels, HMEC-1 cells $[28,33]$.

Treatment of PC3 and HMEC-1 cells with F3-targeted liposomes entrapping anti-PLK1 siRNA produced a significant decrease in cell viability in a lipid concentration-dependent manner, in contrast with the non-targeted counterpart (Fig. 2). These results were likely the consequence of combining in the same technological platform, a component of targeting an internalizing receptor with intracellular triggered release of siRNA [28]. Moreover, targeting angiogenesis, in addition to cancer cells, might be of high importance as tumor growth and metastasis formation are highly dependent on the angiogenic process [34]. 
(A)

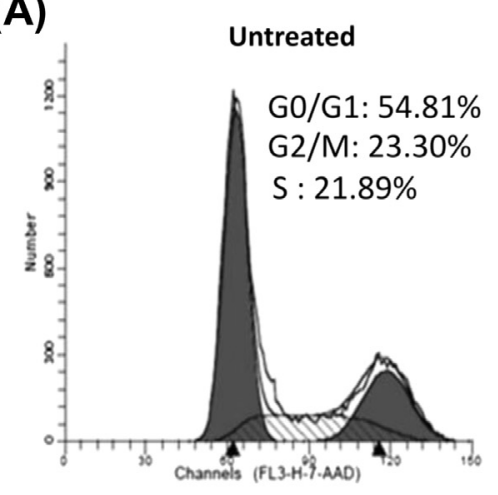

(D)

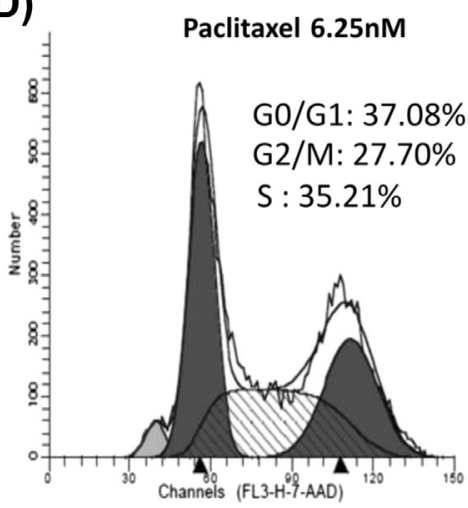

(B)

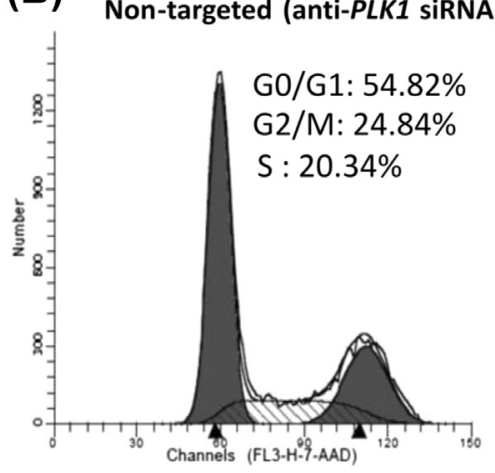

(E) Non-targeted (anti-PLK1 siRNA) +

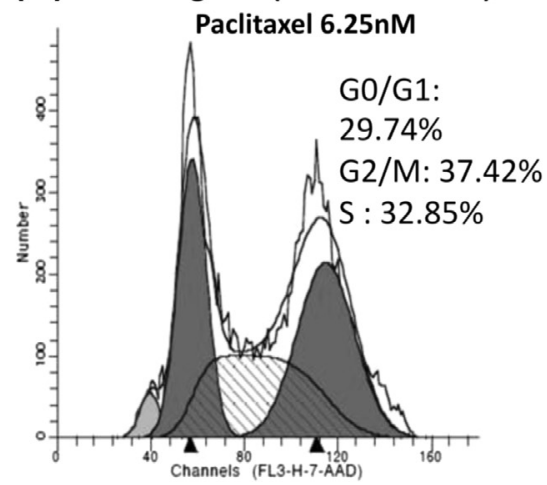

(C)

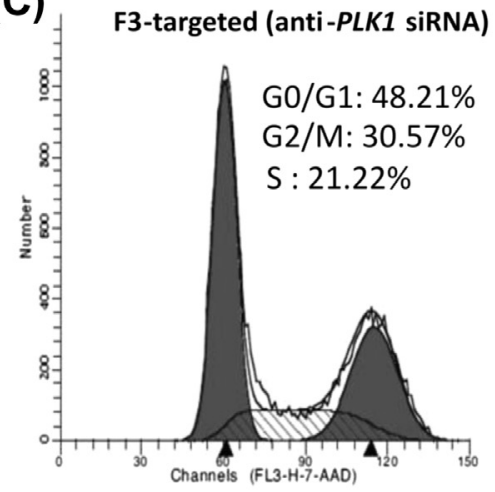

(F)
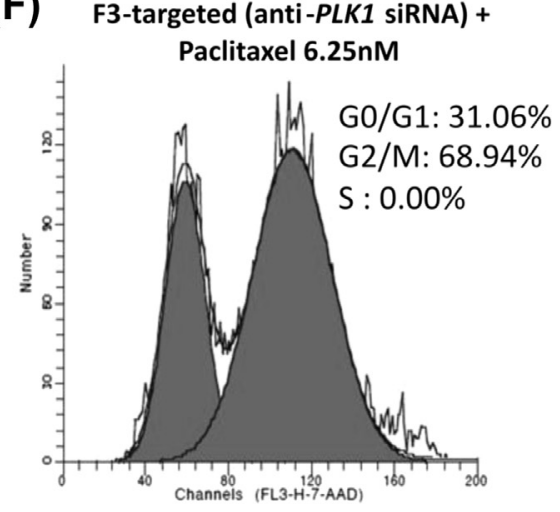

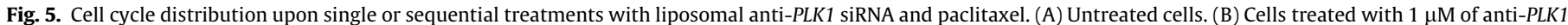

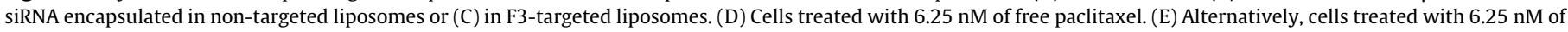

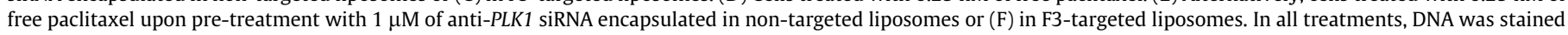
with 7-AAD and cell cycle analyzed by flow cytometry. The results shown are from a representative experiment.

The obtained results are in accordance with other in vitro studies reporting decrease in cancer cell viability upon siRNA-mediated Plk1 downregulation. However, most of those studies have used positively charged transfection reagents to mediate the siRNA delivery into the target cells $[7,8,11,18,35]$, which represents a valuable strategy for in vitro purposes but not for in vivo applications [36]. In fact, upon intravenous administration, positively charged nanoparticles interact with the negatively charged serum proteins forming aggregates that are mainly accumulated in the lungs, liver, and spleen [37]. Moreover, cationic liposomes often induce severe pulmonary toxicity [36]. For these reasons, the in vivo use of this class of nanoparticles has been limited to local administration. In the work of Nogawa et al. [22], intravesical administration of an anti-PLK1 siRNA, complexed with cationic liposomes, effectively prevented the growth of bladder cancer in an orthotopic mouse model. However, this strategy is not feasible when a well established tumor with distant metastasis has to be treated.

To gain further insights into the cellular effects of F3-targeted liposomes entrapping anti-PLK1 siRNA, PLK1 mRNA and protein levels were evaluated. Downregulation at these two levels was achieved only when anti-PLK1 siRNA was delivered by the F3-targeted liposomes, but neither by the non-targeted counterpart nor by the control siRNA delivered by the former (Fig. 3). These observations indicated that the observed decrease in cell viability was correlated with a RNA interference-mediated mechanism.

Although siRNA by itself can bring benefits for cancer treatment, a multi-targeted strategy at the cellular and intracellular level may lead to better clinical outcomes. Furthermore, the use of siRNA to inhibit cancer-relevant molecular targets has been explored as a valuable strategy to enhance chemosensitivity of cancer cells $[9,38,39]$.
Paclitaxel is a microtubule-stabilizing agent leading to mitotic arrest and further cell death, which has been used for the treatment of a wide range of tumors including prostate cancer. Previous reports $[7,8]$ have shown that treatment of breast cancer cells with siRNA targeting PLK1, delivered by cationic reagents, improved cell sensitivity to paclitaxel, in a synergistic or additive manner, depending on the cell line. Therefore, combination of drugs targeting cell cycle, which act by different mechanisms, would be expected to produce significant clinical benefits. This has led us to assess the effect of combining F3-targeted liposomes encapsulating anti-PLK1 siRNA and free paclitaxel on the viability of PC3 prostate cancer cells.

Pre-treatment of cancer cells with anti-PLK1 siRNA encapsulated in F3-targeted liposomes followed by treatment with free paclitaxel led to a significant decrease in $\mathrm{IC}_{50}$ of paclitaxel, from $9.07 \pm 1.58$ to $2.82 \pm 0.58 \mathrm{nM}$ (Table 1 ). The mechanism underlying this sensitization effect was proposed by Spankuch et al. [7], who hypothesized that Plk1 downregulation stabilized the attachment of kinetochores to microtubules, contributing to an imbalance of the spindle checkpoint, which increased the sensitivity to microtubule-interfering drugs like paclitaxel. Moreover, as Plk1 has numerous substrates that are involved in mitosis entry and progression as well as in microtubule dynamics, DNA replication, chromosome dynamics and p53 regulation [40], it was expected that Plk1 downregulation would lead to changes in the expression of those regulators which together also contributed to render cancer cells more prone to the cytotoxic effect of paclitaxel.

Furthermore, cell cycle studies demonstrated that pre-treatment of cells with F3-targeted liposomes encapsulating anti-PLK1 siRNA strongly potentiated the effect of paclitaxel since most cells accumulated in the $\mathrm{G} 2 / \mathrm{M}$ arrest, thus leading to subsequent cell 
death (Fig. 5F). These studies demonstrated that the F3-targeted liposomes, and in contrast to the non-targeted counterpart, could be used to effectively deliver an anti-PLK1 siRNA and sensitize prostate cancer cells to paclitaxel.

\section{Conclusion}

In conclusion, the previously developed F3-targeted liposomes, when encapsulating a siRNA against PLK1, had an important impact on the cell viability of two sub-populations of a solid tumor: the cancer cells and the endothelial cells from angiogenic tumor blood vessels. Therefore, this multi-targeted strategy may constitute a novel and valuable approach for the treatment of prostate cancer. Moreover, its ability to sensitize cancer cells to paclitaxel is of high importance as it can contribute to lower side effects and resistance of cancer cells to paclitaxel. In addition, it is important to emphasize that the relevance of this strategy is not limited to prostate cancer. Different reports have demonstrated that Plk1 downregulation has an anti-proliferative effect on cancer cell lines of different histological origins $[7,8,11,18,35]$. Alongside, our group has observed that F3-targeted liposomes can be specifically uptaken by several cancer cell lines of distinct origin, such as breast (hormone-dependent and triple negative subtypes), colon, and small cell and non-small cell lung cancer [41]. Therefore, it is anticipated that the F3-targeted strategy herein described for Plk1 downregulation can be successfully explored to tumors of diverse histological origin.

\section{Acknowledgements}

Lígia C. Gomes-da-Silva is student of the international PhD program in Experimental Biology and Biomedicine at the Center for Neuroscience and Cell Biology, University of Coimbra and recipient of fellowship from the Portuguese Foundation for Science and Technology (FCT) (ref.: SFRH/BD/33184/2007). The work was supported by the Portugal-Spain capacitation program in Nanoscience and Nanotechnology (ref.: NANO/NMed-AT/0042/2007) and by Grant PEst-C/SAU/LA0001/2011.

\section{References}

[1] A. Jemal, F. Bray, M.M. Center, J. Ferlay, E. Ward, D. Forman, Global cancer statistics, CA Cancer J. Clin. 61 (2011) 69-90.

[2] S.H. Lang, F.M. Frame, A.T. Collins, Prostate cancer stem cells, J. Pathol. 217 (2009) 299-306.

[3] Y. Gan, M.G. Wientjes, J.L. Au, Expression of basic fibroblast growth factor correlates with resistance to paclitaxel in human patient tumors, Pharm. Res. 23 (2006) 1324-1331.

[4] G. Ploussard, B. Paule, L. Salomon, Y. Allory, S. Terry, D. Vordos, A. Hoznek, F. Vacherot, C.C. Abbou, S. Culine, A. de la Taille, Pilot trial of adjuvant paclitaxel plus androgen deprivation for patients with high-risk prostate cancer after radical prostatectomy: results on toxicity, side effects and quality-of-life, Prostate Cancer Prostatic Dis. 13 (2010) 97-101.

[5] D. Hanahan, R.A. Weinberg, The hallmarks of cancer, Cell 100 (2000) 57-70.

[6] D. Hanahan, R.A. Weinberg, Hallmarks of cancer: the next generation, Cell 144 (2011) 646-674.

[7] B. Spankuch, S. Heim, E. Kurunci-Csacsko, C. Lindenau, J. Yuan, M. Kaufmann, K. Strebhardt, Down-regulation of Polo-like kinase 1 elevates drug sensitivity of breast cancer cells in vitro and in vivo, Cancer Res. 66 (2006) 5836-5846.

[8] B. Spankuch, E. Kurunci-Csacsko, M. Kaufmann, K. Strebhardt, Rational combinations of siRNAs targeting Plk1 with breast cancer drugs, Oncogene 26 (2007) 5793-5807.

[9] D.S. Benoit, S.M. Henry, A.D. Shubin, A.S. Hoffman, P.S. Stayton, PH-responsive polymeric sirna carriers sensitize multidrug resistant ovarian cancer cells to doxorubicin via knockdown of polo-like kinase 1, Mol. Pharm. 7 (2010) 442455.

[10] K. Strebhardt, A. Ullrich, Targeting polo-like kinase 1 for cancer therapy, Nat. Rev. Cancer 6 (2006) 321-330.

[11] S. Reagan-Shaw, N. Ahmad, Silencing of polo-like kinase (Plk) 1 via siRNA causes induction of apoptosis and impairment of mitosis machinery in human prostate cancer cells: implications for the treatment of prostate cancer, FASEB J. 19 (2005) 611-613.
[12] W. Weichert, G. Kristiansen, K.J. Winzer, M. Schmidt, V. Gekeler, A. Noske, B.M. Muller, S. Niesporek, M. Dietel, C. Denkert, Polo-like kinase isoforms in breast cancer: expression patterns and prognostic implications, Virchows Arch. 446 (2005) 442-450.

[13] W. Weichert, G. Kristiansen, M. Schmidt, V. Gekeler, A. Noske, S. Niesporek, M. Dietel, C. Denkert, Polo-like kinase 1 expression is a prognostic factor in human colon cancer, World J. Gastroenterol. 11 (2005) 5644-5650.

[14] Y.B. Feng, D.C. Lin, Z.Z. Shi, X.C. Wang, X.M. Shen, Y. Zhang, X.L. Du, M.L. Luo, X. Xu, Y.L. Han, Y. Cai, Z.Q. Zhang, Q.M. Zhan, M.R. Wang, Overexpression of PLK1 is associated with poor survival by inhibiting apoptosis via enhancement of survivin level in esophageal squamous cell carcinoma, Int. J. Cancer 124 (2009) $578-588$.

[15] Y. Qian, E. Hua, K. Bisht, S. Woditschka, K.W. Skordos, D.J. Liewehr, S.M. Steinberg, E. Brogi, M.M. Akram, J.K. Killian, D.C. Edelman, M. Pineda, S. Scurci, Y.Y. Degenhardt, S. Laquerre, T.A. Lampkin, P.S. Meltzer, K. Camphausen, P.S. Steeg, D. Palmieri, Inhibition of Polo-like kinase 1 prevents the growth of metastatic breast cancer cells in the brain, Clin. Exp. Metastasis (2011).

[16] D.P. Han, J.T. Cui, A.G. Lu, X.H. Chen, B. Feng, Y.P. Zong, S. Qu, Q.F. Cao, M.H. Zheng, Influence of silencing Polo-like kinase 1 on migration and invasion of colorectal cancer cells, Zhonghua Wei Chang Wai Ke Za Zhi 14 (2011) 61-64.

[17] G.J. Kops, B.A. Weaver, D.W. Cleveland, On the road to cancer: aneuploidy and the mitotic checkpoint, Nat. Rev. Cancer 5 (2005) 773-785.

[18] B. Spankuch-Schmitt, J. Bereiter-Hahn, M. Kaufmann, K. Strebhardt, Effect of RNA silencing of polo-like kinase-1 (PLK1) on apoptosis and spindle formation in human cancer cells, J. Natl. Cancer Inst. 94 (2002) 1863-1877.

[19] W.J. Xu, S. Zhang, Y. Yang, N. Zhang, W. Wang, S.Y. Liu, H.W. Tian, L. Dai, Q. Xie, X. Zhao, Y.Q. Wei, H.X. Deng, Efficient inhibition of human colorectal carcinoma growth by RNA interference targeting polo-like kinase 1 in vitro and in vivo, Cancer Biother. Radiopharm. 26 (2011) 427-436.

[20] R. Guan, P. Tapang, J.D. Leverson, D. Albert, V.L. Giranda, Y. Luo, Small interfering RNA-mediated Polo-like kinase 1 depletion preferentially reduces the survival of p53-defective, oncogenic transformed cells and inhibits tumor growth in animals, Cancer Res. 65 (2005) 2698-2704.

[21] A.D. Judge, M. Robbins, I. Tavakoli, J. Levi, L. Hu, A. Fronda, E. Ambegia, K. McClintock, I. MacLachlan, Confirming the RNAi-mediated mechanism of action of siRNA-based cancer therapeutics in mice, J. Clin. Invest. 119 (2009) 661-673.

[22] M. Nogawa, T. Yuasa, S. Kimura, M. Tanaka, J. Kuroda, K. Sato, A. Yokota, H. Segawa, Y. Toda, S. Kageyama, T. Yoshiki, Y. Okada, T. Maekawa, Intravesical administration of small interfering RNA targeting PLK-1 successfully prevents the growth of bladder cancer, J. Clin. Invest. 115 (2005) 978-985.

[23] A. Fire, S. Xu, M.K. Montgomery, S.A. Kostas, S.E. Driver, C.C. Mello, Potent and specific genetic interference by double-stranded RNA in Caenorhabditis elegans, Nature 391 (1998) 806-811.

[24] S.M. Elbashir, W. Lendeckel, T. Tuschl, RNA interference is mediated by 21 - and 22-nucleotide RNAs, Genes Dev. 15 (2001) 188-200.

[25] D. Castanotto, J.J. Rossi, The promises and pitfalls of RNA-interference-based therapeutics, Nature 457 (2009) 426-433.

[26] J.N. Moreira, A. Santos, V. Moura, M.C. Pedroso de Lima, S. Simoes, Non-viral lipid-based nanoparticles for targeted cancer systemic gene silencing, J. Nanosci. Nanotechnol. 8 (2008) 2187-2204.

[27] L.C. Gomes-da-Silva, N.A. Fonseca, V. Moura, M.C. Pedroso de Lima, S. Simoes, J.N. Moreira, Lipid-based nanoparticles for siRNA delivery in cancer therapy: paradigms and challenges, Acc. Chem. Res. 45 (2012) 1163-1171.

[28] L.C. Gomes-da-Silva, Y. Fernandez, I. Abasolo, S. Schwartz, J.S. Ramalho, M.C. Pedroso de Lima, S. Simoes, J.N. Moreira, Efficient intracellular delivery of siRNA with a safe multitargeted lipid-based nanoplatform, Nanomedicine (Lond.) (2013).

[29] K. Porkka, P. Laakkonen, J.A. Hoffman, M. Bernasconi, E. Ruoslahti, A fragment of the HMGN2 protein homes to the nuclei of tumor cells and tumor endothelial cells in vivo, Proc. Natl. Acad. Sci. USA 99 (2002) 74447449.

[30] J.N. Moreira, T. Ishida, R. Gaspar, T.M. Allen, Use of the post-insertion technique to insert peptide ligands into pre-formed stealth liposomes with retention of binding activity and cytotoxicity, Pharm. Res. 19 (2002) 265269.

[31] M.E. Kleinman, K. Yamada, A. Takeda, V. Chandrasekaran, M. Nozaki, J.Z. Baffi, R.J. Albuquerque, S. Yamasaki, M. Itaya, Y. Pan, B. Appukuttan, D. Gibbs, Z. Yang, K. Kariko, B.K. Ambati, T.A. Wilgus, L.A. DiPietro, E. Sakurai, K. Zhang, J.R. Smith, E.W. Taylor, J. Ambati, Sequence- and target-independent angiogenesis suppression by siRNA via TLR3, Nature 452 (2008) 591-597.

[32] W.G. Cho, R.J. Albuquerque, M.E. Kleinman, V. Tarallo, A. Greco, M. Nozaki, M.G. Green, J.Z. Baffi, B.K. Ambati, M. De Falco, J.S. Alexander, A. Brunetti, S. De Falco, J. Ambati, Small interfering RNA-induced TLR3 activation inhibits blood and lymphatic vessel growth, Proc. Natl. Acad. Sci. USA 106 (2009) 7137-7142.

[33] L.C. Gomes-da-Silva, A.O. Santos, L.M. Bimbo, V. Moura, J.S. Ramalho, M.C. Pedroso de Lima, S. Simoes, J.N. Moreira, Toward a siRNA-containing nanoparticle targeted to breast cancer cells and the tumor microenvironment, Int. J. Pharm. 434 (2012) 9-19.

[34] A. Abdollahi, J. Folkman, Evading tumor evasion: current concepts and perspectives of anti-angiogenic cancer therapy, Drug Resist. Updat. 13 (2010) 16-28.

[35] B. Spankuch-Schmitt, G. Wolf, C. Solbach, S. Loibl, R. Knecht, M. Stegmuller, G. von Minckwitz, M. Kaufmann, K. Strebhardt, Downregulation of human pololike kinase activity by antisense oligonucleotides induces growth inhibition in cancer cells, Oncogene 21 (2002) 3162-3171. 
[36] S. Simoes, A. Filipe, H. Faneca, M. Mano, N. Penacho, N. Duzgunes, M.P. de Lima, Cationic liposomes for gene delivery, Expert Opin. Drug Deliv. 2 (2005) 237-254

[37] M. Mikhaylova, I. Stasinopoulos, Y. Kato, D. Artemov, Z.M. Bhujwalla, Imaging of cationic multifunctional liposome-mediated delivery of COX-2 siRNA Cancer Gene Ther. 16 (2009) 217-226.

[38] L.S. Mendonca, J.N. Moreira, M.C. de Lima, S. Simoes, Co-encapsulation of antiBCR-ABL siRNA and imatinib mesylate in transferrin receptor-targeted sterically stabilized liposomes for chronic myeloid leukemia treatment, Biotechnol. Bioeng. 107 (2010) 884-893.
[39] S. Trabulo, A.M. Cardoso, T. Santos-Ferreira, A.L. Cardoso, S. Simoes, M.C. Pedroso de Lima, Survivin silencing as a promising strategy to enhance the sensitivity of cancer cells to chemotherapeutic agents, Mol. Pharm. 8 (2011) $1120-1131$.

[40] X.S. Liu, B. Song, X. Liu, The substrates of Plk1, beyond the functions in mitosis, Prot. Cell 1 (2010) 999-1010.

[41] V. Moura, Combining targeted and triggered drug delivery to tumor cells and the tumor microenvironment a novel strategy on cancer therapeutics, in: Faculty of Pharmacy, University of Coimbra, 2010, p. 153. 